\title{
Prediction of cellulose nanofibril (CNF) amount of CNF/polypropylene composite using near infrared spectroscopy
}

\author{
Kazushige Murayama ${ }^{1 *}$ (D) Hikaru Kobori ${ }^{2}$, Yoichi Kojima ${ }^{2}$, Kenji Aoki ${ }^{2}$ and Shigehiko Suzuki ${ }^{3}$
}

\begin{abstract}
The final goal of this study is to establish a classification method of cellulose nanofibril (CNF)/plastic composites such as their CNF amount, CNF types, and resin types, which are expected to progress the commercialization in the future, using near infrared (NIR) spectroscopy. To achieve this goal, NIR spectra of injection and film samples with different types and addition ratios of CNFs in CNF/polypropylene (PP) composites were measured and analyzed in the range of 1000-2200 $\mathrm{nm}$. The results of the principal component analysis using all samples suggest that CNF addition ratio and sample shape could be expressed by principal component (PC) 1 and PC2 scores, which relate to the chemical components of PP and CNF complexly. Furthermore, the partial least-squares (PLS) regression model was able to predict the CNF addition ratio with about 2.0\% accuracy, regardless of CNF type and sample shape. To develop an easier model compared to the PLS model, it was calculated to the simple linear regression model, which used the absorbance quotient of optimum wavelengths combination (OWC). Although this model did not have the accuracy to use the quality control, it is able to discriminate CNF addition ratio of CNF/PP composites with almost the same accuracy as the PLS model. However, if it is possible to separate the sample shapes before the analysis, it is suggested that the OWC regression model is able to predict CNF addition ratio of CNF/PP composites with less than 1\% accuracy.
\end{abstract}

Keywords: Cellulose nanofibril/polypropylene composite, Cellulose nanofibril amount, Near infrared spectroscopy, Quantitative analysis

\section{Introduction}

The production of cellulose nanofibril (CNF)/plastic composites gains attention due to their better physical and mechanical properties [1-3]. Furthermore, CNF has some benefit compared to other fibers to use plastic composites such as biodegradability and renewability. The global market of nanocellulose-added composite materials including CNF/plastic composites was 390.8 metric ton (MT) in 2020 and the volume is expected to reach 922.5 MT in 2026 [4]. However, this rapid increase may possibly result in $\mathrm{CNF} /$ plastic composites causing

\footnotetext{
*Correspondence: kazumura039@ffpri.affrc.go.jp

${ }^{1}$ Forestry and Forest Products Research Institute, 1 Matsunosato, Tsukuba, Ibaraki 305-8687, Japan

Full list of author information is available at the end of the article
}

excessive waste in the near future. Therefore, it is necessary to find the effective and efficient recycling method of $\mathrm{CNF} /$ plastic composites. The products of commercial plastics and plastic composites have been successfully recycled from end products such as automobile parts, appliances, and films. Mastellone [5] reported that the recycling methods of plastics could be allocated to four major categories, viz., re-extrusion (primary), mechanical (secondary), chemical (tertiary) and energy recovery (quaternary). In these categories, re-extrusion and mechanical recycling are waste plastic reusing methods without a chemical reaction process. The advantage of the recycled plastics and composites is that they can be easily applied to the same purpose as original products though these methods. It was reported that the plastic composites with cellulose-based materials did not
Springer Open
C The Author(s) 2022. Open Access This article is licensed under a Creative Commons Attribution 4.0 International License, which permits use, sharing, adaptation, distribution and reproduction in any medium or format, as long as you give appropriate credit to the original author(s) and the source, provide a link to the Creative Commons licence, and indicate if changes were made. The images or other third party material in this article are included in the article's Creative Commons licence, unless indicated otherwise in a credit line to the material. If material is not included in the article's Creative Commons licence and your intended use is not permitted by statutory regulation or exceeds the permitted use, you will need to obtain permission directly from the copyright holder. To view a copy of this licence, visit http://creativecommons.org/licenses/by/4.0/. 
decrease physical and mechanical properties after these types of recycling [6,7]. Thus, it is considered that these recycling methods are suitable for $\mathrm{CNF} /$ plastic composites. To conduct these types of recycling effectively, it is required to classify the composites into the smallest possible groups such as groups of resin types and additive types in advance.

Many researchers have reported that the optimum $\mathrm{CNF}$ addition ratio on $\mathrm{CNF} /$ plastic composites varied in each type of physical and mechanical properties. For example, the Young's modulus of these composites increased with increasing CNF addition ratio [8-11]. On the other hand, their elongation and toughness decreased with an increase in CNF addition ratio. In addition, it was reported that there was an optimum CNF addition ratio to maximize the cell density and minimize the cell diameter of $\mathrm{CNF} /$ polypropylene (PP) foam [12]. These properties are also influenced by CNF and the types of plastic; however, these reports suggest that the classification of $\mathrm{CNF} /$ plastic composites in each CNF addition ratio is important when the re-extrusion and mechanical recycling are conducted. Therefore, near infrared (NIR) spectroscopy is focused on this study.

NIR spectroscopy is a non-destructive analytical method based on the reflectance or absorption of electromagnetic radiation at wavelength range of $800-2500 \mathrm{~nm}$ $\left(12,500-4000 \mathrm{~cm}^{-1}\right)$ [13]. Since NIR spectra allow clear discrimination of various organic compounds, NIR spectroscopy is known to be a useful technique for discriminating different types of wood-based materials, plastics and these wastes [14-18]. Therefore, it is considered that NIR spectroscopy would have a potential to evaluate the quantity of $\mathrm{CNF}$ from $\mathrm{CNF} /$ plastic composite. Furthermore, the practical use of $\mathrm{CNF} /$ plastic composite classification using NIR spectroscopy should be easily implemented, because a plastic sorting device using this method has been already used in the industry.

The final goal of this study is to establish a discrimination method of $\mathrm{CNF} /$ plastic composites using NIR spectroscopy. In this study, NIR spectra of CNF/PP composites with various CNF addition ratio were measured and the prediction models for CNF addition ratio were evaluated. We selected PP for the base plastic in this study because PP is one of the most common plastic bases of composite material. Absorption and scattering in the NIR region are strongly affected by the thickness of the material; however, the shapes and thickness of $\mathrm{CNF} /$ plastic composite wastes would not be uniform. Therefore, NIR spectra of CNF/PP composites with two kinds of sample shapes and two types of CNFs were investigated.

\section{Material and methods Materials}

Table 1 shows the detail of sample conditions, viz., sample name, the shape of the sample, CNF type, CNF addition ratio, and the number of samples. In this study, two kinds of CNFs which are the trial products of companies made by mechanical processes (called CNF-A and CNF-B in this study) were prepared to investigate the effect of CNF types. Although there is also a mixture of around $10-100 \mathrm{~nm}$ width and a few $\mu \mathrm{m}$ length CNFs, the raw materials of these CNFs are different. These types of CNFs are defined in ISO/TS 21346:2021 [19]. Before making samples, pellets were made of one type of PP and each of CNFs by Toclas Corporation in Japan. In the pellets with CNF-A, the CNF addition ratio was adjusted to $0,1.0,3.1,5.0,6.2,10$, and $20 \mathrm{wt} \%$. On the other hand, the addition ratios in the pellets with CNF-B were $0,1.0,2.0$, $3.3,5.0,6.5,8.0$, and $10 \mathrm{wt} \%$.

\section{Sample preparation}

Each of the pellets including CNF-A of $0,1.0,3.1$, 5.0, and $6.2 \mathrm{wt} \%$ or CNF-B of $0,1.0,2.0,3.3,5.0,6.5$, 8.0 , and $10 \mathrm{wt} \%$ was molded to an injection sample $(60 \times 10 \times 3 \mathrm{~mm})$ with an injection pressure of $5 \mathrm{MPa}$ at $200{ }^{\circ} \mathrm{C}$ using an injection-molding machine (Babyplast 6/10P, Cronoplast, Italy). In this study, the injection samples made of the pellets with CNF-A and with CNF-B were called Injection-A and Injection-B,

Table 1 The details of sample conditions: sample name, sample shape, cellulose nanofibril (CNF) types, CNF addition ratios, and number of samples

\begin{tabular}{lllll}
\hline Name & Shape $^{\mathbf{a}}$ & CNF type & CNF addition ratio (wt\%) & $\begin{array}{l}\text { Number of replications per CNF } \\
\text { addition ratio }^{\mathbf{b}}\end{array}$ \\
\hline Injection-A & Injection & CNF-A & $0,1.0,3.1,5.0,6.2$ & 20 (calibration set $=13$, test set $=7$ ) \\
Injection-B & Injection & CNF-B & $0,1.0,2.0,3.3,5.0,6.5,8.0,10$ & 3 (calibration set $=2$, test set $=1$ ) \\
Film-A & Film & CNF-A & $0,5.0,10,20$ & 8 (calibration set $=6$, test set $=2)$ \\
\hline
\end{tabular}

${ }^{a}$ The size of injection and film samples was $60 \times 10 \times 3 \mathrm{~mm}$ and $150 \times 10 \times 0.5 \mathrm{~mm}$, respectively

${ }^{b}$ Both CNFs were the type of cellulose nanofibril in ISO/TS 21,346:2021 [19]

'The number of samples for each CNF addition ratio was shown. Within the parenthesis was shown the number of each sample set for quantitative analysis 
respectively. Furthermore, the pellets with CNF-A of $0,5.0,10$ and $20 \mathrm{wt} \%$ were molded to films with size of $0.5 \mathrm{~mm}$ thickness and $130 \mathrm{~mm}$ width at $200{ }^{\circ} \mathrm{C}$ by a T-die extrusion machine (Research Laboratory of Plastics Technology Co., Ltd, Japan). Afterward, these films were cut into $150 \times 10 \times 0.5 \mathrm{~mm}$. The film samples were called Film-A in this study. All samples were conditioned at room temperature over 1 week prior to the NIR measurement.

\section{Measurement of NIR spectra}

NIR diffuse reflectance spectra were measured using a spectrophotometer (UV-3600 Plus, Shimadzu Co. Ltd, Japan). The NIR spectra were obtained at $2 \mathrm{~nm}$ intervals over the wavelengths from 1000 to $2200 \mathrm{~nm}$. Three scans were repeated and averaged in each sample.

\section{Data analysis}

To remove the scattering effect due to the difference of sample thickness, standard normal variate (SNV) was performed. Each sample of Injection-A, Injection$\mathrm{B}$, and Film-A were randomly split into the calibration and test sets. The number of each sample set for analysis is shown in Table 1.

A principal component analysis (PCA) was performed for SNV spectra of the calibration set to verify the spectral variation and sample parameters such as sample shape, CNF type, and CNF addition ratio. The data were analyzed in the mean-centered states before PCA.

After that, the partial least-squares (PLS) regression analysis of CNF addition ratio was performed on the basis of the mean-centered SNV spectral data of calibration set. Prediction accuracy of the PLS model was evaluated by full cross-validation and external test set.

In addition, the absorption quotient of all wavelengths in each sample was calculated using the raw spectra $\left(t_{i j}\right)$ as in the following equation:

$$
t_{i j}=\frac{\lambda_{i}}{\lambda_{j}}
$$

where $\lambda_{i}$ and $\lambda_{j}$ are the absorbances at $i$ and $j \mathrm{~nm}$, respectively.

The highest regression coefficient $(R)$ between $t_{i j}$ and $\mathrm{CNF}$ addition ratio in CNF/PP composites was searched and then the linear regression model to predict CNF addition ratio was established using the $t_{i j}$ at the highest $R$. We defined this model as the optimum wavelength combination (OWC) regression model in this study. The OWC model was constructed to develop an easier model compared to the PLS model.

\section{Results and discussion}

\section{Raw and SNV NIR spectra}

Figure 1 shows the raw NIR spectra of each calibration set of CNF/PP composites. The baseline of all samples decreased with an increase in the CNF addition ratio, due to the increase of scattering by the CNF as a scattering medium. In film samples under the same CNF addition ratios, uneven absorbances were observed under the lower $10 \mathrm{wt} \% \mathrm{CNF}$ addition ratios, especially in $1800-2200 \mathrm{~nm}$ wavelength, may be due to the non-uniform thickness of the sample edge caused in the cutting process. The variation in thickness was clearly shown in the low CNF addition ratio samples. In all samples, the strong peaks of second overtone $\mathrm{CH}$ symmetric stretching vibration (about $1210 \mathrm{~nm}$ ) and the first overtone $\mathrm{CH}$ degenerate stretching vibration (about $1720 \mathrm{~nm}$ ) were observed. The peak of the $\mathrm{CH}$ combined vibration of stretching and deformation (about $1410 \mathrm{~nm}$ ) was also observed; however, this peak became weak and broad in Film-A with $20 \mathrm{wt} \% \mathrm{CNF}$ addition ratio (Fig. 1c). These peaks were mainly assigned to the $\mathrm{CH} 2$ and $\mathrm{CH} 3$ groups in PP. Furthermore, a weak and broad peak increase occurred at about $2110 \mathrm{~nm}$ according to the increasing $\mathrm{CNF}$ addition ratio, attributed to the $\mathrm{OH}$ combined vibration of stretching and deformation derived from CNF.

Figure 2 shows the NIR spectra of each calibration set of CNF/PP composites after SNV treatment. After SNV treatment, the baseline became almost uniform regardless of CNF addition ratio. The SNV treatment reduced the variability between film samples with the same CNF addition rates ( 0 and $5 \mathrm{wt} \%$ ) observed in the raw spectra (Figs. 1c, 2c). On the other hand, some films with 5 and $10 \mathrm{wt} \% \mathrm{CNF}$ showed similar absorbance values. It is thought that the diffuse reflection by NIR ray decreases in thinner samples. It may also be strongly affected by topical CNF aggregation. In addition to the chemical peaks shown in raw NIR spectra, a weak and broad peak was observed around $1490 \mathrm{~nm}$ (intermolecular and intramolecular hydrogen bonds in CNF) and about $1930 \mathrm{~nm}$ $(\mathrm{OH}$ combination vibration of asymmetric stretching and deformation band) in SNV-NIR spectra. According to increase in $\mathrm{CNF}$ addition ratio, the peaks derived from PP decreased, whereas these from CNF increased. On comparing the sample shapes, the spectral shapes of Injection-A and B were almost the same (Fig. 2a, b). In this study, the base plastic and sample shapes were the same in all injection samples. Thus, it is natural that the absorption of Injection-A and B without CNF is almost the same. A difference in absorbance of NIR between Infection-A and Infection-B including different kinds of CNFs was also not observed. This result suggests that CNF kinds do not affect the CNF/PP composites on the 

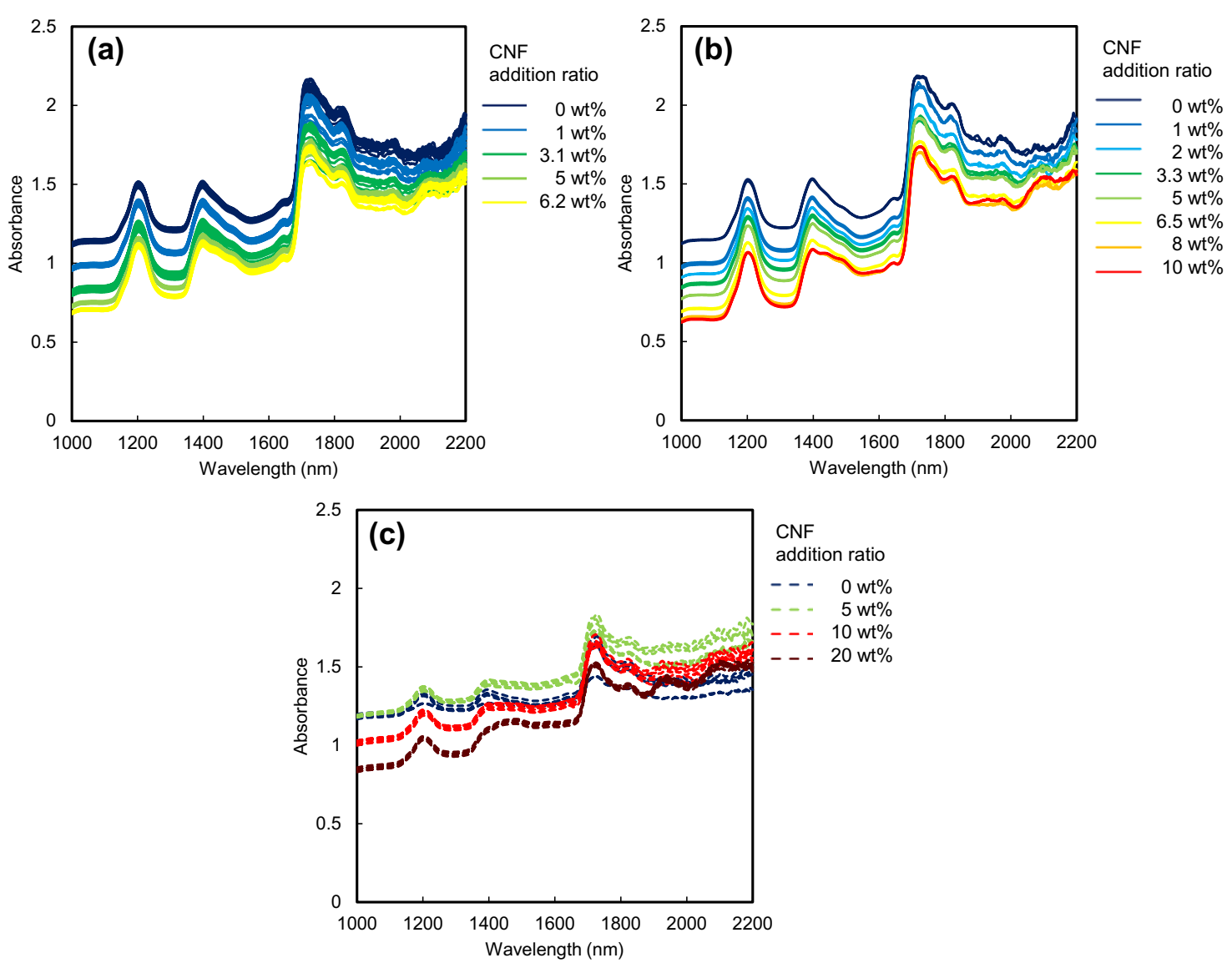

Fig. 1 The raw near infrared (NIR) spectra of each calibration set of CNF/polypropylene (PP) composites: a Injection-A, b Injection-B, c Film-A. The details of sample conditions are shown in Table 1

NIR spectra. The base material of these CNFs was different; however, their size and might not be significantly different in this study. CNF has significantly different size and shape types, such as TEMPO-oxidized CNF [20]. This type of CNF is prepared by the chemical modification process and has $3-4 \mathrm{~nm}$ width and the characteristic functional groups on the outer surface of the fibril. It is called individualized CNF in ISO/TS 21346:2021 [19]. Hence, it will be necessary to confirm the spectra of composites using these types of CNFs for clarification of the effect of CNF types in the future. Furthermore, the absorbance changes of films (Fig. 2c) were smaller than those of injections (Fig. 2a, b). This is the effect of the sample thickness, which can be explained by LambertBeer law.

\section{PCA}

Figure 3 shows the result of PCA for SNV-NIR spectra with all CNF/PP composites. PC1 and PC2 described $79 \%$ and $14 \%$ of data variance, respectively. Therefore, $93 \%$ of the existing variances in all the studied spectra can be captured by two PC. As shown in Fig. 3a, the $\mathrm{CNF}$ addition ratio increased with an increase in the PC1 score with all samples; however, the score was different between the injection and film samples with the same CNF addition ratio. The PC2 score identified the CNF addition ratio, though it was not remarkable compared to the $\mathrm{PC} 1$ score. Furthermore, a difference in the PC2 score was observed between the sample shapes.

In the loading plot of PC1 and PC2 (Fig. 3b), PC1 showed the highest positive loading around $2100 \mathrm{~nm}$ and the highest negative loading at $1760 \mathrm{~nm}$. PC2 showed the highest positive loading at $1730 \mathrm{~nm}$ and the highest negative loading at $1930 \mathrm{~nm}$. These wavelengths derived from chemical bonds of PP and CNF can be observed in the SNV-NIR spectra except for $1760 \mathrm{~nm}$ (Fig. 2).

The results of PCA suggest that the CNF addition ratio and sample shape could be expressed by PC1 and PC2. With these results, we deemed that it was possible to make the model to predict CNF addition ratio in CNF/ $\mathrm{PP}$ composites. Therefore, linear regression analyses were carried out to develop the prediction model as follows. 

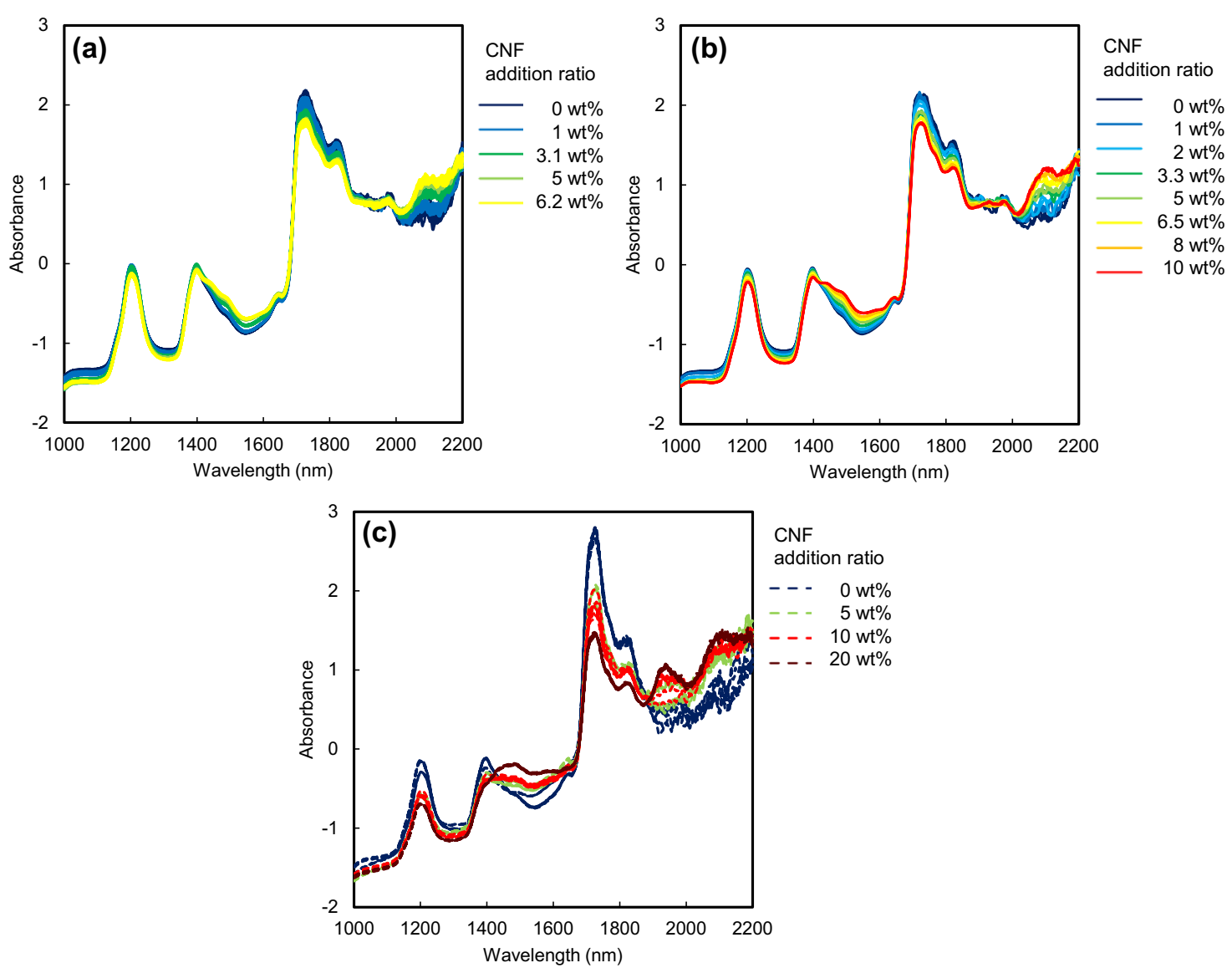

Fig. 2 The NIR spectra of each calibration set of CNF/PP composites after standard normal variate (SNV): a Injection-A, b Injection-B, c Film-A. The details of sample conditions are shown in Table 1
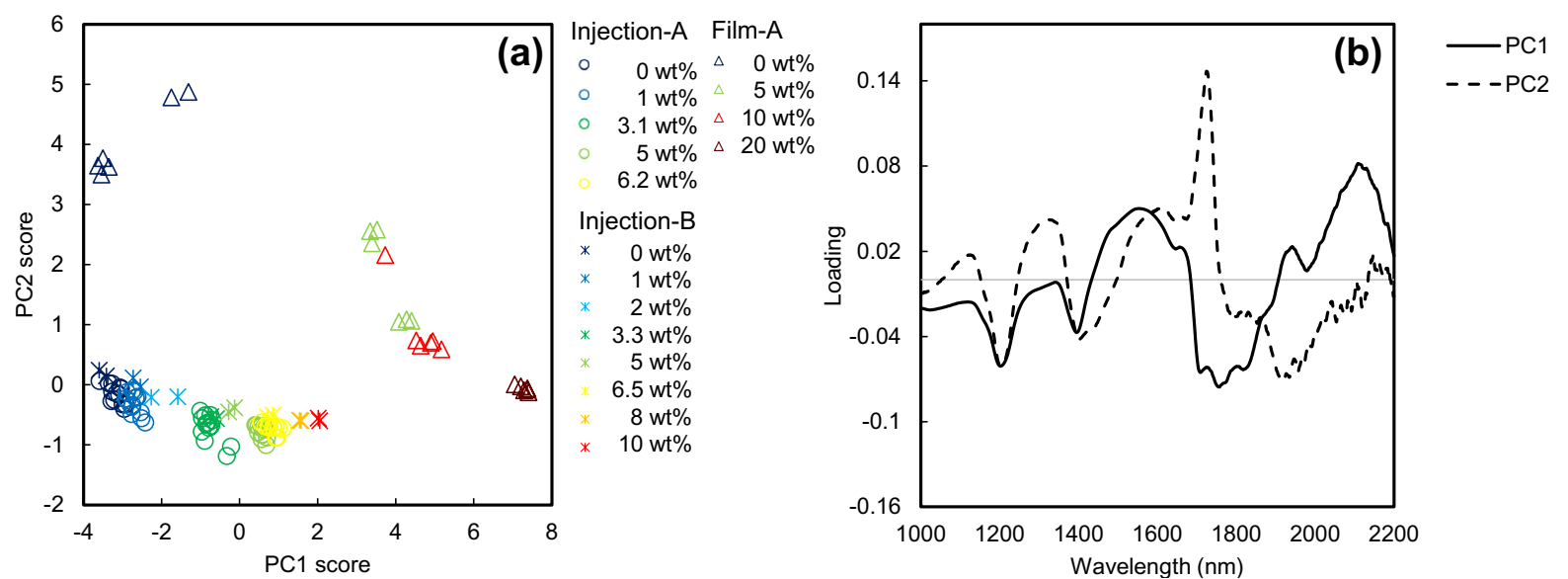

Fig. 3 The result of principal component analysis (PCA) for SNV-NIR spectra with all CNF/PP composites: a principal component (PC)1 and PC2 score plots, $\mathbf{b}$ loading plots of PC1 and PC2. The details of sample conditions are shown in Table 1 


\section{PLS regression}

The results of the PLS regression model for estimating CNF addition ratio using SNV-NIR spectra are shown in Table 2. It is required to configure an appropriate number of factors which show the same coefficient of determination $\left(R^{2}\right)$, standard error (SE), and the ratio of performance to standard deviation (RPD) in calibration and test sets. Furthermore, it is needed to avoid the overfitting caused. However, this model resulted in a little overfitting, may be due to the small number of samples for Injection-B and Film-A. Furthermore, it may be related to no difference of SNV-NIR spectra of some films with $5 \mathrm{wt} \%$ and $10 \mathrm{wt} \%$ CNF (Fig. 2c). In this study, the optimum number of factors, where $R^{2}, \mathrm{SE}$ and RPD did not deviate significantly, was three. In this case, the RPD of cross-validation $\left(\mathrm{RPD}_{\mathrm{V}}\right)$ and prediction $\left(\mathrm{RPD}_{\mathrm{P}}\right)$ were 5.42 and 6.55, respectively. When RPD exceeds five, the model can be used for quality control in general. Even this model has the problem of overfitting; however, the prediction accuracy is sufficiently adapted to quality control of CNF content. Furthermore, a 95\% reliable section is double of standard deviation $(\mathrm{SE} / \sqrt{ } 2)$, which was 1.26 for the calibration set $\left(\mathrm{SE}_{\mathrm{CV}}\right)$ and 0.93 for the test set $\left(\mathrm{SE}_{\mathrm{P}}\right)$. Thus, it was assumed that this model could predict CNF addition ratio with about $2.0 \mathrm{wt} \%$ accuracy in this study.

Figure 4 shows the regression plot of the optimum PLS regression models. The regression coefficient showed a high positive value at 1480 and $1930 \mathrm{~nm}$ and a high negative value at 1850 and $2200 \mathrm{~nm}$. A lot of wavelengths of the high regression values were similar in wavelengths of the high PCA loading of the PC1 and PC2 scores (Fig. 3), especially the $1930 \mathrm{~nm}$ showed the highest value of regression and $\mathrm{PC} 2$ values.

\section{OWC regression}

The PLS regression model in this study was able to predict the CNF addition ratio with about $2.0 \mathrm{wt} \% \mathrm{CNF}$ accuracy regardless of CNF type and sample thickness. However, to apply the method to actual production line, it is desirable to establish a simple estimation method which can compute the CNF addition ratio with simple data with narrow wavelength range. It was reported that it was possible to estimate the wood flour amount in wood/plastic composite with the quotients of wood

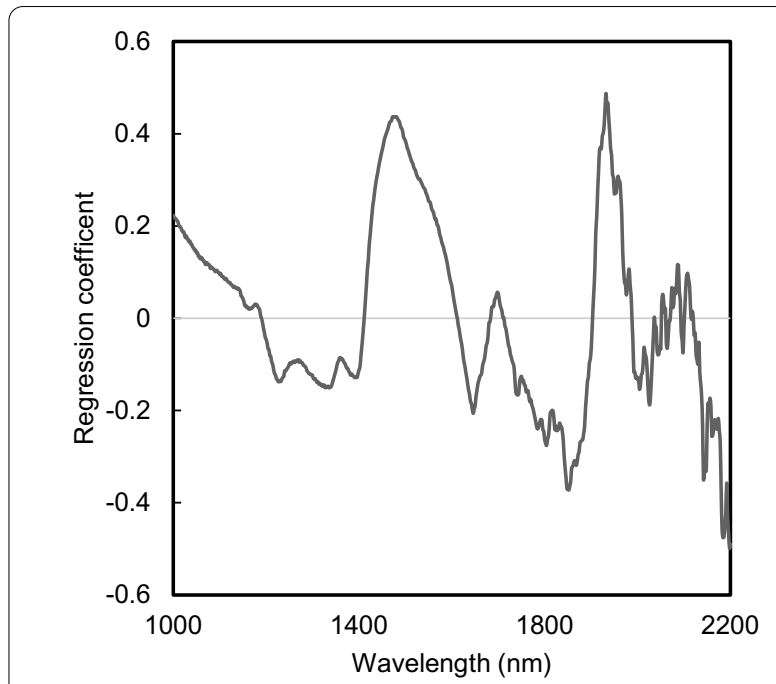

Fig. 4 The regression plot of the optimum PLS regression models

and polyethylene infrared absorption peaks [21]. It is considered that a similar method can be used for CNF/ PP composite quantification in this study because of the presence of PP and CNF absorption peaks in the NIR spectra. However, a lot of peaks related to PP and CNF were observed in the NIR spectra in this study. Hence, we calculated the absorbance quotient of all wavelength combinations. Then, the linear regression model, viz., OWC regression model, was established using the best wavelength combination using raw NIR spectra. Here, the OWC models were constructed in each of the sample shape as well as the model established using all sample data.

Figure 5 shows the 2D images of $R$ between CNF addition ratio and the raw absorbance quotients of all wavelengths combination. In the 2D image of all samples, wavelengths of 1200,1400 , and $1700-2100 \mathrm{~nm}$ were used for one of the wavelength combinations, resulting in high $R$ (Fig. 5a). On the other hand, the $R$ of the higher 0.8 was found in many combinations by the sample shape classified models (Fig. 5b, c).

The results of the OWC regression models of each sample shape are shown in Table 3. $R^{2}, \mathrm{SE}_{\mathrm{P}}$, and $\mathrm{RPD}_{\mathrm{P}}$ of the OWC model of all samples were $0.93,1.11$, and

Table 2 The results of the partial least-squares (PLS) regression model for estimating CNF addition ratio using SNV-NIR spectra

\begin{tabular}{|c|c|c|c|c|c|c|c|}
\hline \multirow[t]{2}{*}{ Data } & \multicolumn{4}{|c|}{ Calibration set } & \multicolumn{3}{|c|}{ Test set } \\
\hline & Factor & $R^{2}$ & $\mathrm{SE}_{\mathrm{CV}}$ & $\mathrm{RPD}_{\mathrm{v}}$ & $R^{2}$ & $\mathrm{SE}_{\mathrm{p}}$ & $\mathrm{RPD}_{\mathrm{P}}$ \\
\hline CNF addition ratio (wt \%) & 3 & 0.96 & 0.89 & 5.42 & 0.97 & 0.66 & 6.55 \\
\hline
\end{tabular}

Factor: the optimum number of PLS factors, $R^{2}$ : coefficient of determination, $\mathrm{SE}_{\mathrm{CV}}$ : standard error of cross-validation, $\mathrm{RPD}$ : ratio of performance to standard deviation of cross-validation, $\mathrm{SE}_{\mathrm{p}}$ : standard error of prediction, $\mathrm{RPD}_{\mathrm{p}}$ : ratio of performance to standard deviation of prediction 


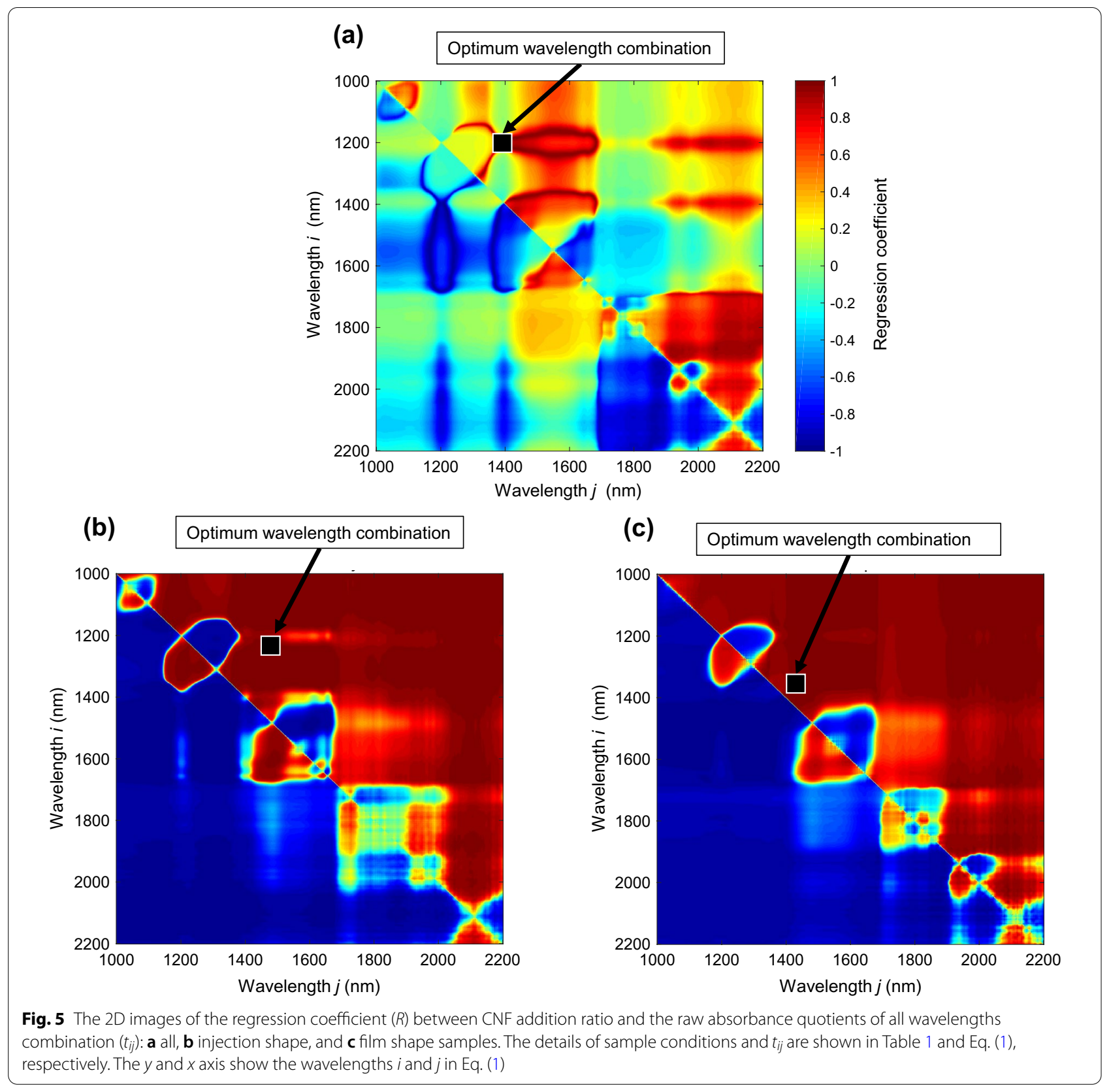

Table 3 The result of optimal wavelength combination (OWC) regression models for each sample shape

\begin{tabular}{|c|c|c|c|c|c|c|c|c|c|}
\hline \multirow{2}{*}{$\begin{array}{l}\text { CNF addition ratio } \\
\text { (\%) }\end{array}$} & \multicolumn{5}{|c|}{ Calibration set } & \multicolumn{4}{|c|}{ Test set } \\
\hline & owc & $N$ & $R^{2}$ & $\mathrm{SE}_{\mathrm{M}}$ & $\mathrm{RPD}_{\mathrm{M}}$ & $\mathrm{N}$ & $R^{2}$ & $\mathrm{SE}_{\mathrm{p}}$ & $\mathrm{RPD}_{\mathrm{P}}$ \\
\hline All & $1418 / 1220$ & 105 & 0.97 & 0.84 & 5.74 & 51 & 0.93 & 1.11 & 3.87 \\
\hline Injection & $1518 / 1310$ & 81 & 0.99 & 0.10 & 26.5 & 43 & 0.99 & 0.11 & 24.7 \\
\hline Film & $1468 / 1362$ & 24 & 0.99 & 0.29 & 25.8 & 8 & 0.99 & 0.44 & 17.8 \\
\hline
\end{tabular}

OWC optimal wavelength combination, $N$ number of samples, $R^{2}$ coefficient of determination, $S E_{M}$ standard error of OWC model, RPD ${ }_{M}$; ratio of performance to standard deviation of OWC model, $S E_{p}$ standard error of prediction, $R P D_{p}$ ratio of performance to standard deviation of prediction 
3.87 , respectively. Because the RPD value is from 2.5 to 5 , the model cannot be applied to the quality control in product line; however, it has a certain accuracy. Compared to the PLS model (Table 2), the RPD value was lower, whereas overfitting did not occur. Furthermore, because a $95 \%$ reliable section of this model was \pm 1.57 , it was able to estimate a CNF addition ratio with about $2 \%$ accuracy as well as the PLS model. In the model of injection shape samples, $R^{2}, \mathrm{SE}_{\mathrm{P}}$, and $\mathrm{RPD}_{\mathrm{P}}$ were $0.99,0.11$, and 24.7 , respectively. On the other hand, in the model of film shape samples, $R^{2}, \mathrm{SE}_{\mathrm{P}}$, and $\mathrm{RPD}_{\mathrm{P}}$ were $0.99,0.44$, and 17.8, respectively. Although the sample number of films was not enough, it is assumed that the OWC model of the film could predict CNF addition ratio with less than $1 \mathrm{wt} \%$ CNF accuracy, because the $95 \%$ reliable section of the film samples model was 0.62 in the test set in this study. Therefore, if it is possible to classify the sample shapes before the analysis, and the OWC model can predict CNF addition ratio of CNF/PP composites of less than $1 \%$ accuracy.

The OWCs in injection, film, and combined sample models were 1518/1310, 1468/1362, and 1418/1220 nm, respectively. Looking at the optimal wavelengths of all the sample models, $1418 \mathrm{~nm}$ appeared between the combination of the $\mathrm{CH}$ stretching vibration of $1410 \mathrm{~nm}$ and the $\mathrm{CH}$ deformation vibration and the associated $\mathrm{OH}$ $(1435-1480 \mathrm{~nm})$. The other $(1210 \mathrm{~nm})$ was the second overtone of the $\mathrm{CH}$ symmetric stretching vibration. The all sample models obtained the highest $R^{2}$ for the combination of the peaks related to CNF and PP as expected. The OWC in the injection sample model was observed in the combination of the wavelengths of CNF-derived absorption band (1500-1595 nm, intermolecular hydrogen bonding, associated $\mathrm{OH}$ and without absorption band. The film samples model was found in the combination of wavelengths of CNF-derived absorption band $(1435-1480 \mathrm{~nm})$ and no absorption band. In the wavelengths without chemical bands (1310, $1362 \mathrm{~nm}$ ), the baseline significantly varied due to change in CNF addition ratio (Fig. 2). It is thought that nonchemical band wavelength became the best-contributed wavelength on these models to separate sample shape, because the effect of variation of NIR ray inside the sample is enhanced.

\section{Conclusion}

This study aimed to propose an effective and efficient method to predict CNF content of CNF/PP composites using NIR spectroscopy. Therefore, the effect of CNF type, sample shape and CNF addition ratio on NIR spectra was evaluated. The results of PCA suggest that CNF addition ratio and sample shape could be expressed by the PC1 and PC2 scores, which are related to the chemical components of PP and CNF complexly. Furthermore, the PLS regression model was able to predict the CNF addition ratio with about $2.0 \%$ accuracy regardless of CNF type and sample shape in this study. Furthermore, even though the OWC regression model of all samples is not sufficient for quality control, it has the same level of sorting accuracy as the PLS regression model. Thus, it is found that the OWC linear regression drawn through all sample data can be used to develop a simpler prediction model with similar prediction accuracy of the PLS model. If the sample shapes can be classified before the analysis, the OWC model is able to predict CNF addition ratio of CNF/PP composites with a better accuracy than $1 \mathrm{wt} \%$.

They are useful results to predict the CNF addition ratio of CNF/PP composites which have higher added value than other plastics effectively and efficiently. In future studies, it is required to evaluate the effect of sample shapes, surface roughness, CNF types, CNF aggregation, CNF dispersion, and resin types and these combinations for putting into use.

\section{Abbreviations}

CNF: Cellulose nanofibril; NIR: Near infrared; PP: Polypropylene; PCA: Principal component analysis; PC: Principal component; PLS: Partial least squares; OWC: Optimum wavelength combination; MT: Metric ton; SNV: Standard normal variate; CNF-A/CNF-B: Cellulose nanofibril-type CNFs which are defined in ISO/ TS 21346:2021 and they are different products; Injection-A: Injection molded samples with CNF-A added PP composites; Injection-B: Injection molded samples with CNF-B added PP composites; Film-A: Film shaped samples with CNF-A added PP composites; $t_{i j}$ : The absorption quotient of all wavelengths in each sample was calculated using the raw spectra; $\lambda_{i} / \lambda_{j}$. The absorbances at $i$ and $j \mathrm{~nm}$; $R$ : Regression coefficient; $R^{2}$ : Coefficient of determination; SE: Standard error; $\mathrm{SE}_{\mathrm{CV}}$ : $\mathrm{SE}$ of cross-validation; $\mathrm{SE}_{\mathrm{p}}$ : $\mathrm{SE}$ of test set; $\mathrm{SE}_{\mathrm{M}}$ : Standard error of OWC regression model; RPD: The ratio of performance to standard deviation; RPD $D_{V}$ RPD of cross-validation; RPD : RPD of prediction; RPDM: Ratio of performance to standard deviation of the OWC regression model.

\section{Acknowledgements \\ None.}

\section{Authors' contributions}

The first and corresponding author participated sufficiently in the work to take public responsibility for the entire contents of the manuscript. The co-authors have participated sufficiently in the work to take public responsibility for part of the contents of the manuscript. All authors read and approved the final manuscript.

\section{Funding}

None.

Availability of data and materials Not applicable.

\section{Declarations}

Ethics approval and consent to participate Not applicable.

\section{Consent for publication}

Not applicable.

Competing interests

The authors declare that they have no competing interests. 


\section{Author details}

${ }^{1}$ Forestry and Forest Products Research Institute, 1 Matsunosato, Tsukuba, Ibaraki 305-8687, Japan. ${ }^{2}$ Faculty of Agriculture, Shizuoka University, 836 Ohya, Suruga-ku, Shizuoka 422-8529, Japan. ${ }^{3}$ Shizuoka Professional University of Agriculture, 678-1 Tomigaoka, Iwata-ku, Shizuoka 438-8577, Japan.

Received: 1 October 2021 Accepted: 12 January 2022

Published online: 22 January 2022

\section{References}

1. Kobe R, Iwamoto S, Endo T, Yoshitani K, Teramoto Y (2016) Stretchable composite hydrogels incorporating modified cellulose nanofiber with dispersibility and polymerizability: mechanical property control and nanofiber orientation. Polymer 97:480-486. https://doi.org/10.1016/j. polymer.2016.05.065

2. Yano H (2007) New developments in bio-based materials (in Japanese). CMC Publishing Co., Ltd., Tokyo, pp 63-70

3. Yano H, Nakahara S (2004) Bio-composites produced from plant microfiber bundles with a nanometer unit web-like network. J Mater Sci 39:1635-1638

4. Global nanocellulose market research report 2020 (2020) QYResearch Group. Beijing, China.

5. Mastellone ML (1999) Thermal treatments of plastic wastes by means of fluidized bed reactors. Dissertation, Second University of Naples.

6. Senba T (2018) Recyclability of Cellulose Nano Fiber Reinforced Plastic (in Japanese). Seikei-Kakou 30(2):56-58. https://doi.org/10.4325/seikeikakou. 30.56

7. Beg MDH, Pickering KL (2008) Reprocessing of wood fibre reinforced polypropylene composites Part I: Effects on physical and mechanical properties. Composites 39(7):1091-1100. https://doi.org/10.1016/j. compositesa.2008.304.01

8. Oliveira MHG, Maia SHT, Talabi IS, Canto BL, Lucas AA (2020) Characterization of cellulose nano/microfibril reinforced polypropylene composites processed via solid-state shear pulverization. Polym Compos 42(3):13711382. https://doi.org/10.1002/pc.25907

9. Pengo Y, Nair SS, Chen H, Yan N, Cao J (2018) Effects of lignin content on mechanical and thermal properties of polypropylene composites reinforced with micro particles of spray dried cellulose nanofibrils. ACS Sustainable Chem Eng 6(8):11078-11086. https://doi.org/10.1021/acssu schemeng.8b02544

10. Bhasney MS, Kumar A, Katiyar V (2020) Microcrystalline cellulose, polylactic acid and polypropylene biocomposites and its morphological, mechanical, thermal and rheological properties. Compos B. https://doi. org/10.1016/j.compositesb.2019.107717

11. Kahavita NHDK, Samarasekara AMPB, Amarasinghe SAD, Karunanayake $L$ (2019) Influence of surface modification of cellulose nanofibers (CNF) as the reinforcement of polypropylene based composite. In: 2019 Moratuwa Engineering Research Conference (MERCon). http://doi.org/https://doi. org/10.1109/MERCon.2019.8818869.

12. Wang L, Ando M, Kubota M, Ishihara S, Hikima Y, Ohshima M, Sekiguchi T, Sato A, Yano H (2017) Effects of hydrophobic-modified cellulose nanofibers (CNFs) on cell morphology and mechanical properties of high void fraction polypropylene nanocomposite foams. Compos A 98:166-173. https://doi.org/10.1016/j.compositesa.2017.03.028

13. Tsuchikawa S, Yamamoto K, Inoue K (2003) Discriminant analysis of wood-based materials using near-infrared spectroscopy. J Wood Sci 49:275-280

14. Abe H, Kurata Y, Watanabe K, Ishikawa A, Noshiro S, Fujii T, Iwasa M, Kaneko H, Wada H (2020) The separation of softwood and hardwood in historical wooden statues of the Nazenji-temple in Japan using NIR spectroscopy. IAWA J 41(4):740-750. https://doi.org/10.1163/22941932-bja10 038

15. Masoumi H, Safavi MS, Khani Z (2012) Identification and classification of plastic resins using near infrared reflectance spectroscopy. International Scholarly and Scientific Research \& Innovation 6(5):877-884. https://doi. org/10.5281/zenodo.1076916

16. Kobori H, Yonenobu H, Noma J, Tsuchikawa S (2008) Discriminant analyzing system for wood wastes using a visible-near-infrared chemometric imaging technique. Appl Spectrosc 62(8):854-859. https://doi.org/10. 1366/000370208785284295

17. Feldhoff R, Huth-Fehre T, Cammann K (1998) Detection of inorganic wood preservatives on timber by near infrared spectroscopy. J Near Infrared Spectrosc 6(1):A171-A173

18. Feldhoff R, Huth-Fehre T, Kantimm T, Quick L, Broek CK, W, Wienke D, Fuchs H, (1995) Fast identification of packaging waste by near infrared spectroscopy with an InGaAs array spectrograph combined with neural networks. J Near Infrared Spectrosc 3(1):3-9. https://doi.org/10.1255/jnirs. 49

19. ISO/TS 21346:2021 (2021) Nanotechnologies - Characterization of individualized cellulose nanofibril samples. International Organization for Standardization, Switzerland.

20. Isogai A, Saito T, Fukuzumi H (2011) TEMPO-oxidized cellulose nanofibers Nanoscale 3:71-85. https://doi.org/10.1039/CONR00583E

21. Kobayashi M, Kubo S, Kataoka Y, Ishikawa A, Matsunaga M, Kiguchi M, Ohtomo Y (2015) Quantification of wood and plastics in wood plastic composites (WPCs) by means of differential scanning calorimetry and infrared spectroscopy $\|<b r>-$ The applicability to WPCs containing a mixture of PP and PE as raw material- (in Japan). MOKUZAI HOZON (Wood Protection) 41(1):8-17

\section{Publisher's Note}

Springer Nature remains neutral with regard to jurisdictional claims in published maps and institutional affiliations.

\section{Submit your manuscript to a SpringerOpen ${ }^{\circ}$ journal and benefit from:}

- Convenient online submission

- Rigorous peer review

- Open access: articles freely available online

- High visibility within the field

- Retaining the copyright to your article

Submit your next manuscript at $\boldsymbol{\nabla}$ springeropen.com 